\title{
Modulation of Rat Kidney Stone Crystallization and the Relative Oxidative Stress Pathway by Green Tea Polyphenol
}

\author{
Zizhi Li,* Linna Chang, Xiuli Ren,* Yanan $\mathrm{Hu}$, and Zhenhua Chen* \\ Cite This: ACS Omega 2021, 6, 1725-1731 \\ Read Online
}

ABSTRACT: The role of tea polyphenol (TP) in modulating kidney stone crystallization and regulating the relative nephropathy pathway of rats was investigated. Calcium oxalate $(\mathrm{CaOx})$ crystallization and oxidative stress are essential for kidney stone diseases. The kidney stone model in a rat was established by using ethylene glycol to affect the oxalic acid metabolism. The crystallization process of $\mathrm{CaOx}$ in the rat kidney was modulated by different TP intakes. At the same time, the effects of different types of $\mathrm{CaOx}$, extracted from the rat kidney, on the proliferation and differentiation of HK-2 cells were also studied. The results showed that calcium oxalate monohydrate crystals were obtained in the blank control and the low-dose TP groups. However, $\mathrm{CaOx}$

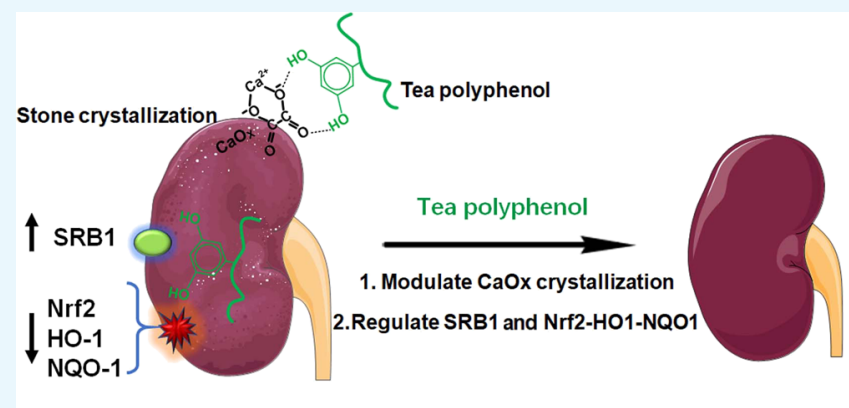
crystals extracted from higher-TP-intake groups were mainly calcium oxalate dihydrate. Moreover, the size of the CaOx crystals produced in TP intake groups was much smaller than that of the blank control group. Cell experiment results show that TP can effectively reduce the damage of $\mathrm{CaOx}$ crystals to $\mathrm{HK}-2$ cells. Further research found that TP can significantly improve oxidative stress in cases of kidney stones. TP has been proven to control $\mathrm{CaOx}$ crystallization in vitro, but the in vivo research results obtained through the rat stone model in this paper are novel and originally important for researching the relationship between tea drinking and preventive treatment of kidney stone diseases.

\section{INTRODUCTION}

Approximately $80 \%$ of kidney stones are composed of calcium oxalate and bring serious problems to people's health. ${ }^{1}$ Although many technologies are developed for treating kidney stones (such as lithotripsy and surgeries), challenges such as high treatment costs and adverse side effects still exist. ${ }^{1}$ In addition to necessary surgical treatment, a preventable method should be adopted. ${ }^{2}$ Therefore, many works that focus on modulating the crystallization of calcium oxalate have been reported in order to develop effective therapeutic and/or preventive agents against stone formation. ${ }^{3-11}$ However, because of the lack of full learning of the calcium oxalate crystallization process and effective dietary, natural, and safe crystallization inhibitors, kidney stones still affect millions of people until now. ${ }^{12}$ Thus, it is urgent to investigate effective calcium oxalate crystallization inhibitors in vivo.

Although green tea polyphenol (TP) has been investigated in biomedical applications recently, ${ }^{13-22}$ usually, green tea is still considered not suitable for recommendation to kidney stone patients. However, our previous work consolidated that green tea extracts can strongly inhibit the formation of calcium oxalate monohydrate (COM) in vitro. ${ }^{5}$ Recent reported work proved the obvious inhibition role of $\mathrm{TP}$ on $\mathrm{CaOx}$ formation. ${ }^{11,23,24}$ Thus, green TP can be used as a good inhibitor for COM according to the above reported work. ${ }^{5,23}$ However, the relative in vivo research on the stone formation inhibition mechanism of green TP is still void. Therefore, to solve this issue, in the present work, the roles of TP in modulating kidney stone crystallization and regulating the relative nephropathy pathway of rats in vivo were investigated. The kidney stone model in a rat was established. The crystallization process of $\mathrm{CaOx}$ in the rat kidney modulated by different TP intakes was investigated. At the same time, the effects of different types of $\mathrm{CaOx}$ on the proliferation and differentiation of HK-2 cells were also studied. TP has been proven to successfully regulate COM crystallization in vitro according to our previous work, ${ }^{5}$ but the in vivo research results obtained through the rat stone model in this paper are novel and originally important for researching the relationship between tea drinking and treatment of kidney stones.

\section{RESULTS AND DISCUSSION}

Photos of rat kidneys in different experimental groups are shown in Figure 1a. Graph 1 (Figure 1a) is one of the classical

Received: December 3, 2020

Accepted: December 30, 2020

Published: January 7, 2021 
a)
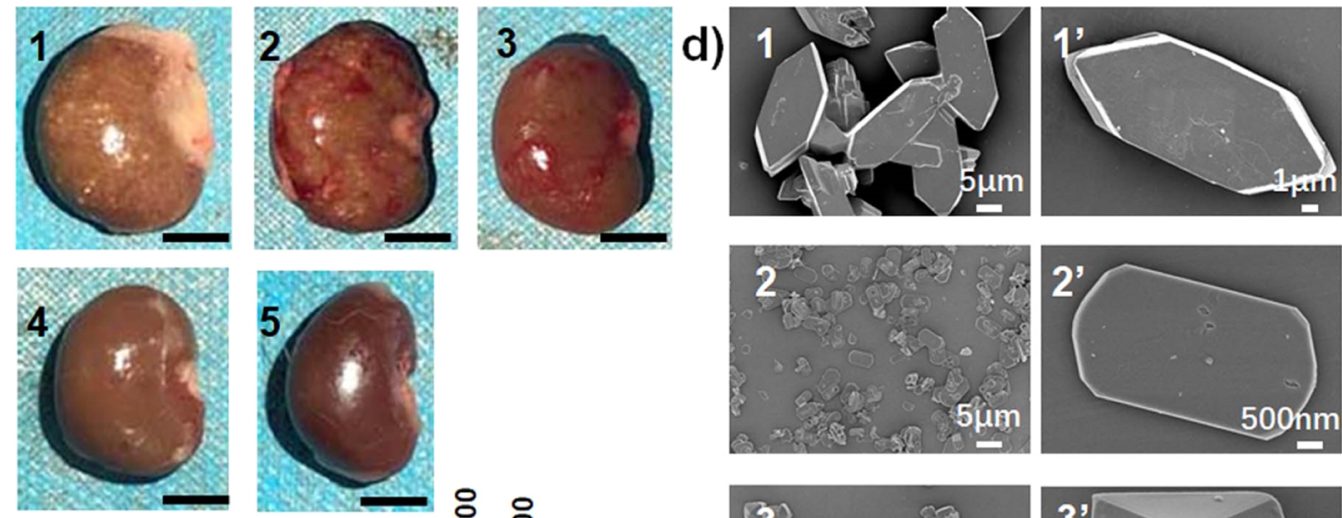

b)
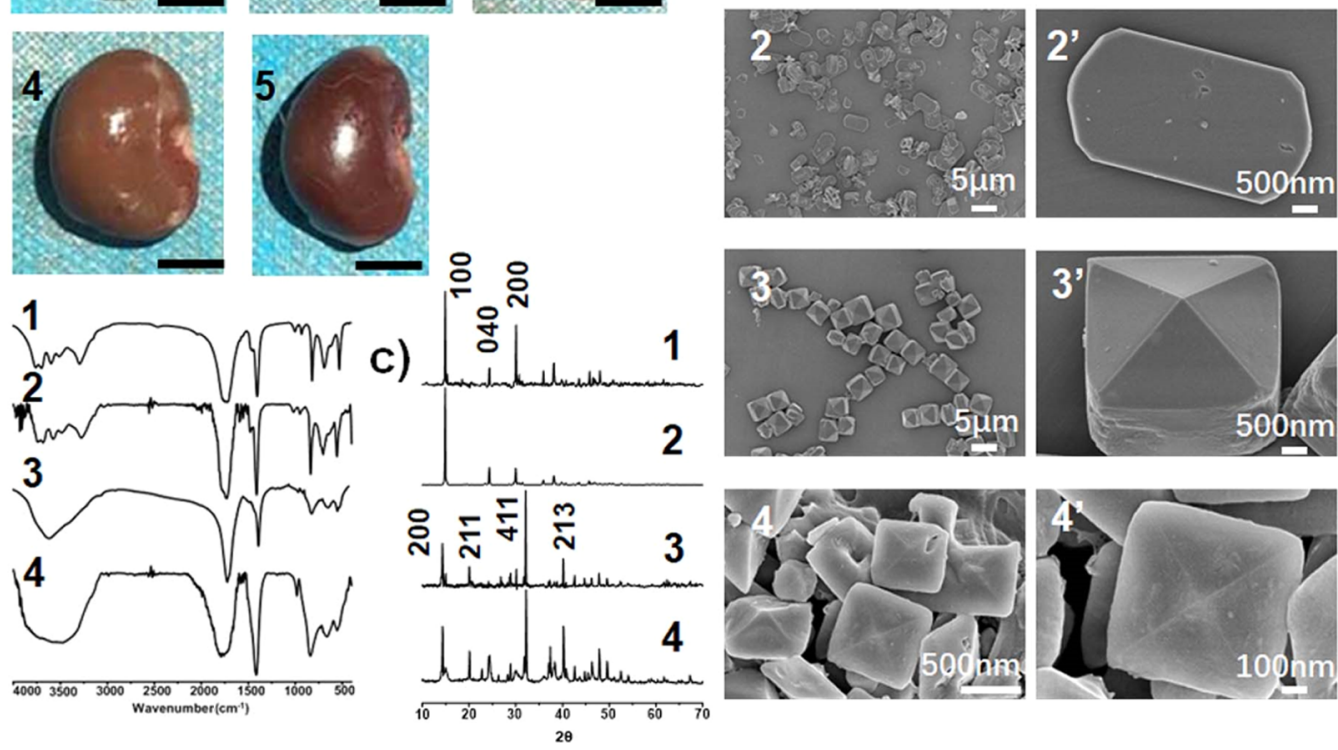

Figure 1. (a) Photos of rat kidneys in different experimental groups: 1, EG groups; 2, EG-TP30; 3, EG-TP100; 4, EG-TP300; and 5, control group (normal kidney); the scales are all $5 \mathrm{~mm}$; photos were taken by "Linna Chang". (b) FTIR spectra of the crystals extracted from groups 1-4. (c) XRD reflections of the crystals extracted from groups 1-4. (d) SEM images of the crystals extracted from groups $1-4$. In (b-d), groups 1 and 2 are ascribed to COM; groups 3 and 4 are ascribed to COD.

kidney images of the rat in the kidney stone model group (EG group). Compared to the normal kidney (control group, graph 5 , Figure 1a), it is obvious that the kidney from the EG group shows abnormal swelling. Also, many white stone crystals can be seen under the kidney epidermis. This suggests that the kidney stone model in the rat was successfully established. In the low-TP-intake group (EG-TP30), the swelling of the kidney (graph 2, Figure 1a) has been alleviated. However, white kidney stone particles can still be discriminated under the kidney epidermis. A further increase of the TP intake amount to $100 \mathrm{mg} / \mathrm{kg}$ rat body weight (EG-TP100) (graph 3, Figure 1a) indicated that the swelling of the kidney had been obviously alleviated. Also, the tiny amount white stone crystals can only be seen on the bottom part of the kidney in Figure 1a3. With the increase of the TP intake amount to $300 \mathrm{mg} / \mathrm{kg}$ rat body weight (EG-TP300), Figure 1a4 shows that the kidney has only light swelling and no observable kidney stone particles. These results might suggest that the increased TP intake amount plays a significant role in reducing kidney swelling and inhibiting stones. Fourier transform infrared (IR) (FTIR) spectroscopy of the calcium oxalate extracted from the kidney in groups $1-4$ is shown in Figure 1a. The IR of the crystals extracted from the EG group (Figure 1b1) and EGTP30 group (Figure 1b2) presents the characteristic bands of COM [5]. However, the FTIR spectra in Figure 1b3,b4 confirm that the crystals extracted from the EG-100 group and EG-TP300 group were COD [5]. X-ray diffraction (XRD) reflections of the crystals extracted from kidneys from groups $1-4$ are shown in Figure 1c. The main reflections of EG and EG-TP30 group crystals are located at $2 \theta=14.88,24.30$, and $29.95^{\circ}$, attributed to the $\{100\},\{040\}$, and $\{200\}$ planes of COM, respectively. The main reflections of EG-TP100 and
EG-TP300 group crystals are located at $2 \theta=14.23,20.01$, 32.10 , and $40.10^{\circ}$, attributed to the $\{200\},\{211\},\{411\}$, and $\{213\}$ planes of calcium oxalate dihydrate (COD), respectively. These IR and XRD results validated that in the model rat kidney (EG group) and the low intake amount of the TP group's rat kidney (EG-TP30), the extract kidney stones are COM. However, in the higher intake amount of the TP group's rat kidney (EG-TP100 and EG-TP300), the extract kidney stones are mainly COD. This in contrast suggests that TP might inhibit the formation of COM and modulate the $\mathrm{CaOx}$ crystallization into COD.

Scanning electron microscopy (SEM) images in Figure 1d further provide the morphology information of the crystals extracted from groups $1-4$. Figure $1 \mathrm{~d} 1, \mathrm{~d} 1^{\prime}$ proves that the crystal extract from the rat kidney in the EG group (model stone group) is COM in the tabular shape. These crystals have dimensions of $20.7 \mu \mathrm{m}$ (length, $\langle 001\rangle) \times 8.1 \mu \mathrm{m}$ (width, $\langle 010\rangle) \times 1.3 \mu \mathrm{m}$ (height, $\langle 100\rangle$ ). In the EG-TP30 group, the crystal extracts from the kidneys are also flat sheet-shaped COM crystals (Figure 1d2). However, these COM crystals are much smaller $(4.6 \mu \mathrm{m} \times 2.5 \mu \mathrm{m} \times 72.4 \mathrm{~nm})$ than those from the EG group. In addition, unlike the sharp apex angles of the COM crystals in the EG group, the apex angles of these COM crystals in the EG-TP30 group are more rounded. These comparisons clearly illustrate the regulating role of TP in controlling the size and shape of COM in vivo.

Unlike COM crystals obtained in the EG group and EGTP30 group, SEM images in Figure 1d3,d3' reveal that the crystals obtained from the EG-TP100 group are classical COD with a flat tetragonal bipyramid shape. These COD crystals were mainly composed of $\{101\}$ planes with a $4.1 \mu \mathrm{m}$ side length and a $0.98 \mu \mathrm{m}$ height along the $c$-axis. However, in the 


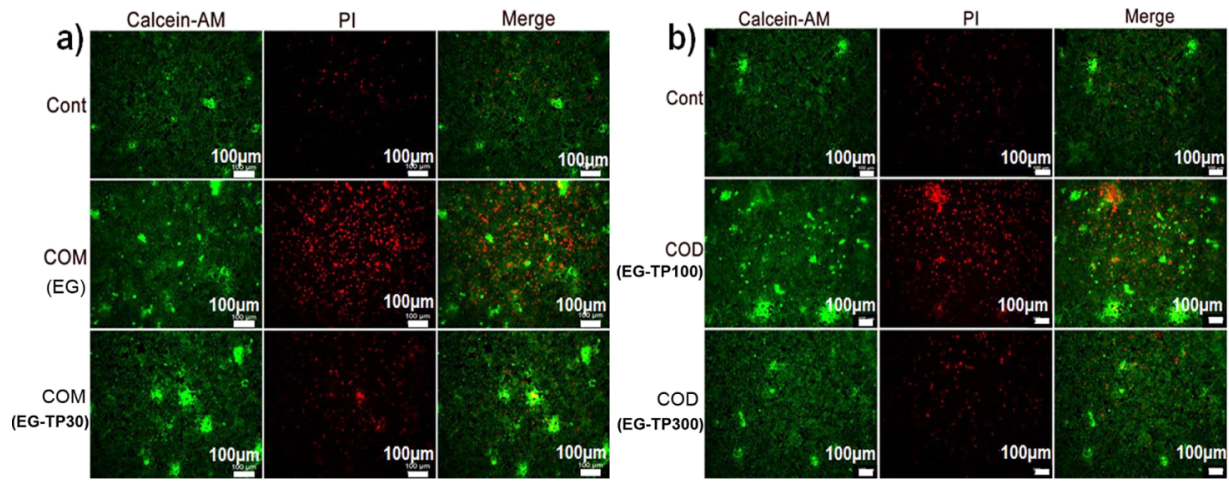

Figure 2. Effects of (a) COM (EG) and COM (EG-TP30) and (b) COD (EG-TP100) and COD (EG-TP300) on HK-2 cell viability; calcein-AM and PI were used to stain HK-2 cells.

EG-TP300 group, the obtained COD crystals are flat octahedrons (Figure 1d4,d4') with an average side length of $670 \mathrm{~nm}$. These nano-sized crystals are much smaller than those in the EG-TP100 group. These differences in SEM images clearly reveal the function of TP in modulating $\mathrm{CaOx}$ crystals from COM to COD and controlling the crystal size.

The above results demonstrated that TP inhibits the formation of COM and preforms COD in vivo. COM is thermodynamically stable and has been found more frequently than COD clinically. ${ }^{5,12,25}$ COM adheres more than COD to epithelial cells in culture. ${ }^{25}$ These pieces of evidence strongly suggest that the transformation from COM to COD is benign for preventing and treating kidney stones, but the mechanism is not clear. Thus, the effect of the obtained $\mathrm{CaOx}$ crystals on the epithelial cells, renal histology, and molecular biology analysis should be further investigated.

The effects of COM (EG), COM(EG-TP30), COD(EGTP100), and COD(EG-TP300) on HK-2 cell viability are shown in Figure 2. Unlike that in the control group (Figure 1a, top row), the propidium iodide (PI) fluorescence intensity of the COM (EG) group increased significantly (Figure 1a, central row), which indicates that when cultured with COM (EG), the cell activity decreased and cell death appeared. However, when cultured with COM from the EG-TP30 group (Figure 1a, bottom row), the apoptosis of these HK-2 cells has been obviously decreased compared to those cultured with COM from the EG group. Figure $1 \mathrm{~b}$ shows the images of cells cultured with COD from the EG-TP100 and EG-TP300 groups. Compared with the COM (EG) group, the fluorescence intensity of PI in Figure $1 \mathrm{~b}$ (central row and bottom row) obviously decreased. After careful discrimination, it could be found that the intensities of PI in COM (EGTP30) and COD (EG-TP100) are almost the same. However, the COD (EG-TP300) group presents a much lower PI intensity. These results suggest that the TP intake modified the obtained $\mathrm{CaOx}$ crystals from COM microcrystals to COD nanocrystals in vivo. When cocultured with HK-2 cells, the TPmodified COD nanocrystals showed much lower toxicity than COM microcrystals.

To better investigate the effects of COD nanocrystals and COM microcrystals on HK-2 cells, confocal laser scanning microscopy (CLSM) images of HK-2 Cells cocultured with $\mathrm{COM}$ and/or $\mathrm{COD}$ were obtained. When without $\mathrm{CaOx}$ (Figure 3a), these HK-2 cells grew well and the cytoskeleton presented a normal spindle structure. When the cells were cultured with COM microcrystals (EG), the CLSM image revealed that many $\mathrm{COM}$ crystals adhered to and/or

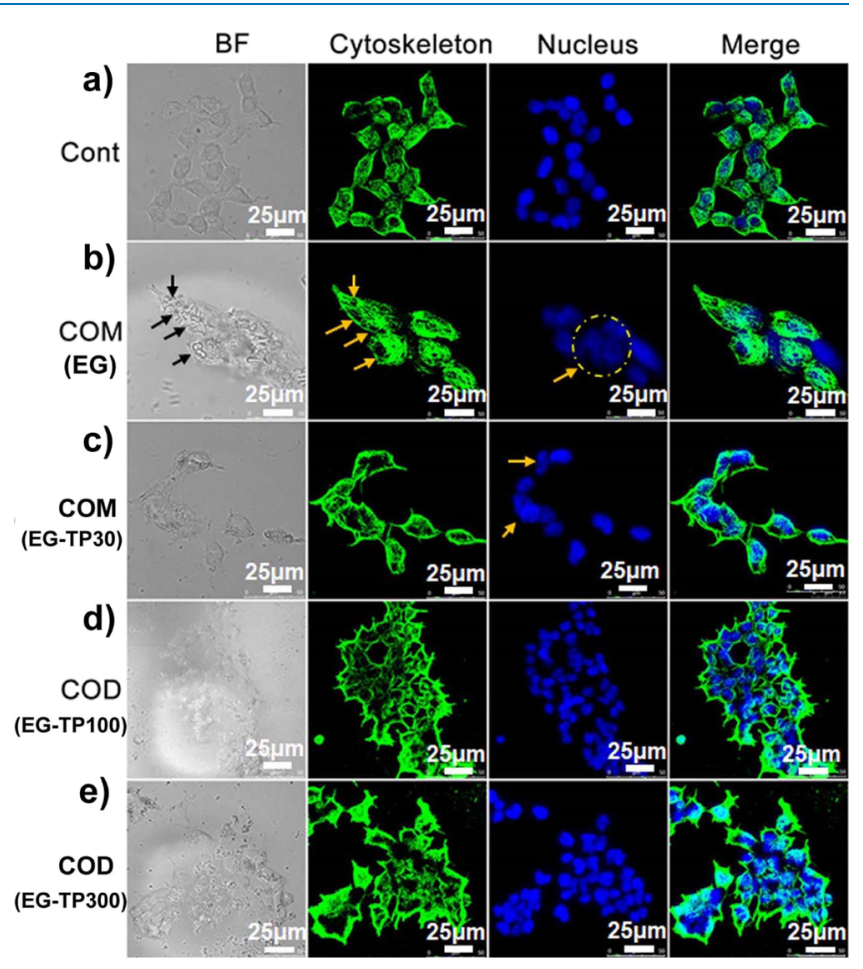

Figure 3. CLSM images of HK-2 cells cocultured in the (a) control group, (b) COM (EG) group, (c) COM (EG-TP30) group, (d) COD (EG-TP100) group, and (e) COD (EG-TP300) group. The cytoskeleton was stained green with tubulin (FITC), and Hoechst 33258 was used to stain nuclei into blue. BF implies bright field.

intervened the cells (indicated by the black arrows, left image in Figure $3 \mathrm{~b}$ ). Yellow arrows in the second image of Figure $3 \mathrm{~b}$ further confirm that these COM crystals damaged the cytoskeleton and left caves on the cells. The third image of Figure $3 \mathrm{~b}$ further indicates the swell and the swelling and fusion of cell nuclei (indicated by the arrow and dashed cycle). Figure $3 \mathrm{c}$ provides the morphology of HK-2 cells cultured with COM from the EG-TP30 group; the swelling of nuclei has been alleviated; however, nuclei fusion (indicated by the arrows) and a few cytoskeleton caves could still be discriminated. Figure $3 \mathrm{~d}$ shows no obvious nuclei swelling but could be observed for the cells cultured with COD from the EG-TP100 group. In addition, these cells in Figure 3d appeared to be increased much in cell density compared to those observed in Figure 3b,c. The images in Figure 3e suggest that the cells cultured with COD (EG-TP300) grew well and 
presented a normal spindle cytoskeleton and no caves could be found on the cells. Furthermore, no swelling nuclei could be observed in Figure 3e. Also, the cell density in Figure 3e is also higher than those in Figure 3b,c.

The above fluorescence microscopy and CLSM results indicate that COM (EG) and COD (EG-TP300) have obviously different effects on HK-2. COM (EG) seriously affected the proliferation of $\mathrm{HK}-2$ and caused apoptosis. However, COD (EG-TP300) did not present obvious toxicity to the renal tubular epithelial cells. To further confirm this different effect on HK-2 cells between COM (EG) and COD (EG-TP300) in vivo, renal histology results are shown in Figure 4. It can be found that the glomerular tubules in the

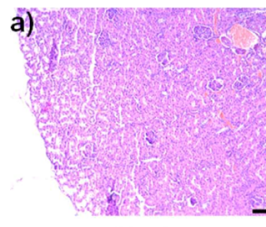

Cont

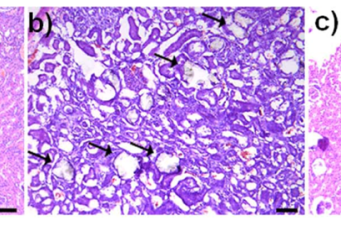

EG c)

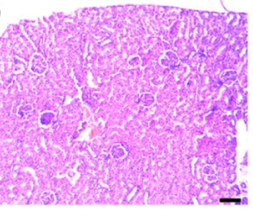

EG-TP300
Figure 4. Renal sections of the (a) control group, (b) EG group, and (c) EG-P300 group were stained with hematoxylin and eosin. The scales were all $100 \mu \mathrm{m}$.

kidney from the control group were in the normal shape (Figure 4a). However, the basic shape of the glomerular glomeruli in the EG group has been damaged and filled with many kidney stones (Figure $4 \mathrm{~b}$, indicated by the arrows). This might suggest that the renal function of the rat in the EG group was severely impaired. Unlike the EG group, the renal histology image in Figure $3 \mathrm{c}$ reveals that the rat kidney in the EG-TP300 group restored the basic shape of the kidney, and the glomerular glomeruli were normal and complete.

The above results indicate that $\mathrm{TP}$ is effective in preferentially promoting the formation of COD over COM in the kidney stone model rat in vivo. In addition, COM from the EG group has serious cytotoxicity to renal tubular epithelial cells. However, COD from the EG-TP300 group did not present obvious cytotoxicity to renal tubular epithelial cells. Furthermore, renal histology results (Figure 4b) suggest that the retained COM crystals damaged the renal tissue structure. However, in the EG-TP300 group, no COD crystals (Figure 4c) could be discriminated and the renal tissue structure is normal and complete. These results and analyses might propose that the TP intake has played a significant role in modulating the crystallization of $\mathrm{CaOx}$ in vivo in the kidney. Also, the molecular biology mechanism about the kidney stone in the model rat might be regulated by TP intake. To elucidate this mechanism, western blot (WB) has been performed on the renal tissues from the control group, EG group, and EG-TP300 group. The results in Figure 5a suggest that Nrf2, HO-1, and NQO-1 were obviously increased in EG, which indicated that the level of oxidative stress in the rat kidney was higher than that in the control group and EG-TP300 group. The analysis results of $\mathrm{Nrf} 2$ (nuclear factor erythroid-2-related factor 2), HO-1 (heme oxygenase-1), and NQO-1 (NADPH quinineoxidoreductase-1) bands shown in Figure $3 \mathrm{~b}-\mathrm{d}$ further confirmed the obvious increased oxidative stress in the EG group. Also, the increase of oxidative stress might injure the membrane of renal tubular epithelial cells. At the same time, as SR-B1 is a lipid metabolism-related protein, the decrease of SR-B1 content is considered to affect the occurrence and development of inflammation. ${ }^{26}$ As shown in Figure 6, after intragastric administration, SR-B1 in the kidney decreased a)

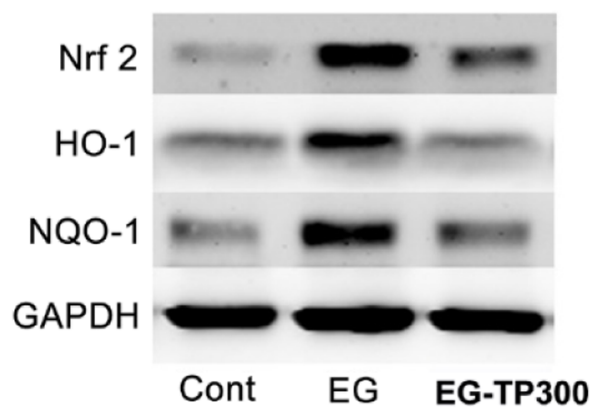

c)

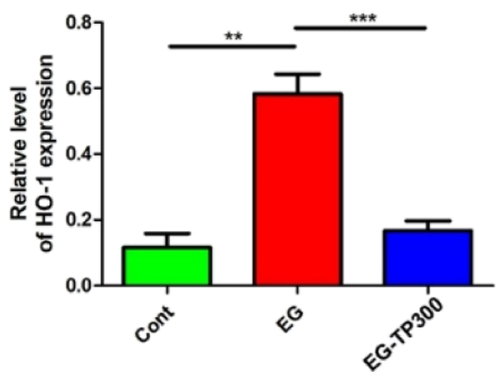

b)

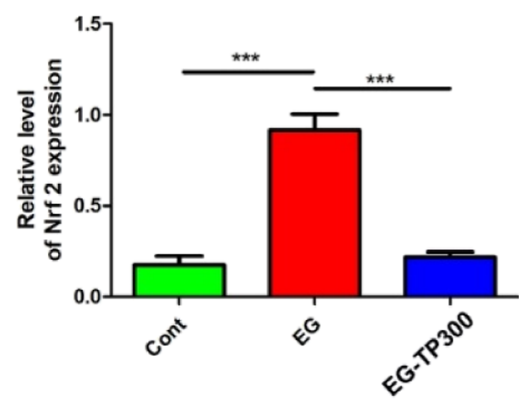

d)

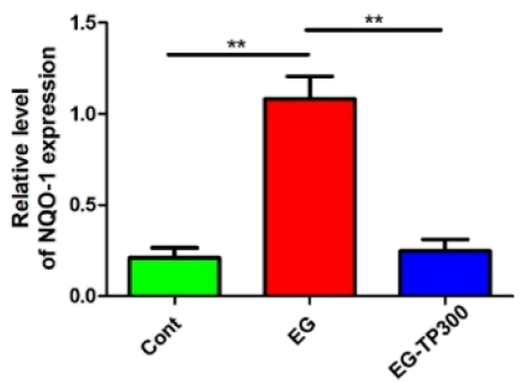

Figure 5. WB results and analysis of the renal tissue from the Cont (control group), EG, and EG-TP300 groups. (a) WB results of different proteins; $(\mathrm{b}-\mathrm{d})$ analysis results of Nrf2, HO-1, and NQO-1 bands $(p<0.05)$. 
a)

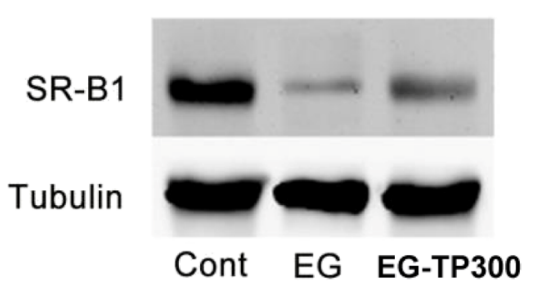

b)

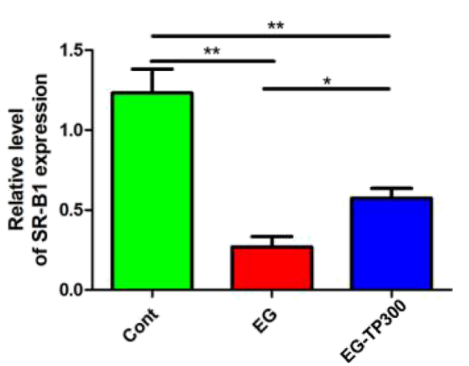

Figure 6. WB results and analysis of the renal tissue from the Cont, EG, and EG-TP300 groups. (a) WB results of SR-B1 and tubulin; (b) analysis of SR-B1 $(p<0.05)$.

significantly. Therefore, the decrease of SR-B1 (Figure 6) may suggest that the increase of oxidative stress in the model rat's kidney may also affect the occurrence and development of inflammation. However, in the EG-TP300 group, the expression of SR-B1 was obviously increased. This evidence strongly suggests that the TP intake played an effective role in anti-inflammation in the stone model rat's kidney.

The above results demonstrate that $\mathrm{TP}$ is benign in prevention and treatment of the kidney stone in the model rat, and the origin of TP's protective and preventive roles is schematically illustrated in Scheme 1. The illustration artwork

Scheme 1. Illustration of TP in Modulating Kidney Stone Crystallization and Regulating the Relative Nephropathy Pathway

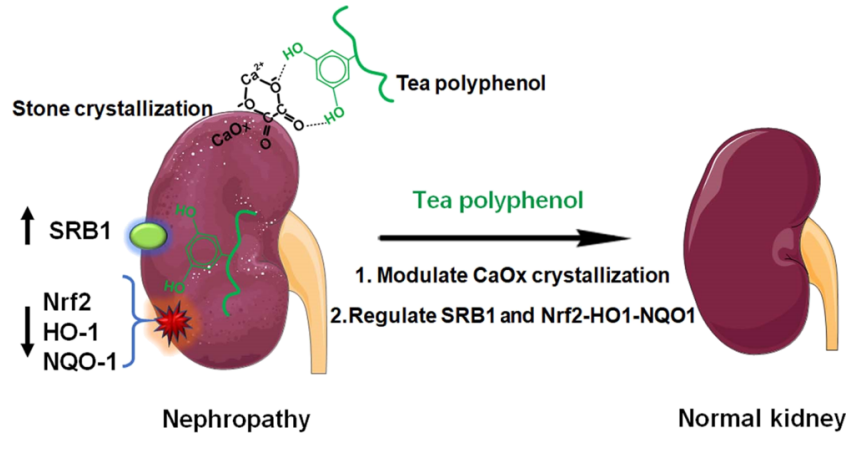

suggests the role of TP in modulating kidney stone crystallization and regulating the relative nephropathy pathway. The mechanisms behind might be explained by the following points: (1) the TP intake plays an important role in inhibiting COM in vivo, preferring COD to COM. This might be because the H-bonding between TP molecules and $\mathrm{CaOx}$ nuclei mediated the crystallization of $\mathrm{CaOx}$ crystals (illustrated in Scheme 1) according to our previous work. ${ }^{5}$ (2) The crystal shape and aggregation in the kidney could affect the attachment of calcium oxalate to renal tubular epithelial cells, which is crucial in stone formation. ${ }^{25}$ (3) COM is the main component of calcium oxalate stones, which cause membrane injury of renal tubular epithelial cells. The administration of TP has been proved to inhibit free radicals induced by COM. The results suggest that $\mathrm{Nrf} 2, \mathrm{HO}-1$, and NQO-1 were significantly decreased by TP administration (Figure 5a), which indicated that the level of oxidative stress in the rat kidney was controlled by the TP intake. At the same time, the obvious increased expression of SR-B1 (Figure 6) is the evidence which strongly suggests that the TP intake played an effective role in anti-inflammation in the stone model rat's kidney. Therefore,
TP has been proved to show preventive and curative effects on the kidney stone.

\section{CONCLUSIONS}

In summary, based on our preliminary work in vitro, we performed the administration of TP in the animal experiment. $\mathrm{TP}$ has been proved to regulate $\mathrm{CaOx}$ crystallization in vivo, favoring the formation of COD over COM. The administration of TP has been proved to inhibit free-radical production induced by COM. The results suggest that Nrf2, HO-1, and NQO-1 have significantly been inhibited by TP administration (Figure 5a), which indicated that the level of oxidative stress in the rat kidney was controlled by the TP intake. At the same time, the obvious increased expression of SR-B1 (Figure 6) is the evidence which strongly suggests that the TP intake played an effective role in anti-inflammation in the stone model rat's kidney. Therefore, TP has been proved to show preventive and curative effects on the kidney stone. These research results obtained through the rat stone model in this paper are novel and originally important for researching the relationship between tea drinking and the prevention and treatment of kidney stones. As far as the scientific community is concerned, we wish that our article will provide some useful data and information on calcium oxalate crystallization and stone disease therapy. Thus, the present strategy may promote its medicinal as well as technological application. We hope that our article will make general readers aware of the fact that a healthy dietary will be very helpful in keeping diseases away. We also hope that more and more people choose green tea as their daily beverage.

\section{MATERIALS AND METHODS}

4.1. Materials. Ethylene glycol (>99\%) and $\mathrm{NH}_{4} \mathrm{Cl}$ $(>99.5 \%)$ were obtained from Sigma Chemical Co. (St Louis, MO, USA). TP ( $\geq 98$ wt \%, Wuxi Taiyo Green Power Co., Ltd., Jiangsu, China; the chemical structure is shown in Figure S1). HK-2 cells were bought from the Chinese Academy of Medical Sciences (Beijing, China).

4.2. Animals and Ethical Approval. Sprague-Dawley rats (SD rats, 12 weeks old, mean body weight $\sim 250 \mathrm{~g}$ ) were used. All the steps of the animal experiment strictly followed the research ethics issued by Jinzhou Medical University.

4.3. Animal Grouping and the Modeling Method. The rats were divided into the blank control group, stone model group, and TP groups. Each group has 10 rats. Except the control group, all groups were given $1 \%$ ethylene glycol tap water and $2 \%$ ammonium chloride $2 \mathrm{~mL} /$ stomach once a day. Meanwhile, the rats in the control group were given distilled water of the same volume by gavage once each day for 4 weeks. 
In the TP groups, according to the various TP intake amounts each day, the groups were divided into the EG-TP30 (30 mg $\mathrm{TP} / \mathrm{kg}$ rat body weight) group; EG-TP100 (10 mg TP/kg rat body weight) group; and EG-TP300 (300 mg TP/kg rat body weight) group. The delivery method of TPs is gastric administration.

4.4. Effects of COM and COD on Cell Viability. To study the effects of COM(EG), COM (EG-TP30), COD(EGTP100), and COD(EG-TP300) on HK-2 cells, $500 \mu \mathrm{g} / \mathrm{mL}$ COM or COD was cocultured with HK-2 cells, and after 1 day, cells were stained with calcein-am/PI and then detected at 535 and $490 \mathrm{~nm}$ by fluorescence microscopy, respectively.

4.5. Immunofluorescence. In each group, HK-2 cells were rinsed three times by phosphate-buffered saline (PBS) after incubation. After that, the cells were stabilized in $4 \%$ paraformaldehyde for $0.5 \mathrm{~h}$. After washing with PBS three times, they were blocked for $2 \mathrm{~h}$ using goat serum (5\%). Then, primary anti-tubulin antibodies were used for staining the incubated cells at $4{ }^{\circ} \mathrm{C}$ for $12 \mathrm{~h}$. After washing by PBS (three times). These cells were incubated with certain secondary antibodies for $1 \mathrm{~h}$ and washed with PBS. At last, the cells were stained by Hoechst 33258, etc.

4.6. WB Analysis. A BCA detection kit (Pierce, IL, USA) was used for detection. The proteins $(4 \mu \mathrm{g} / \mathrm{L}, 200 \mu \mathrm{L})$ were heated at $100{ }^{\circ} \mathrm{C}$ for $10 \mathrm{~min}$ and then separated using $10 \%$ sodium dodecyl sulfate polyacrylamide gel electrophoresis gel. Then, they were placed on polyvinylidene fluoride (PVDF) membranes and treated with Tris-buffered saline with $0.1 \%$ Tween 20 detergent (1\% BSA) for $2 \mathrm{~h}$. The protein was transferred to the PVDF membrane and stabilized overnight with anti-rabbit SR-B1, HO-1, Nrf2, NQO-1 antibodies, antimouse tubulin, and glyceraldehyde 3-phosphate dehydrogenase $(\mathrm{GAPDH})$ at $4{ }^{\circ} \mathrm{C}$. After that, the related secondary antibody was added and treated for $2 \mathrm{~h}$. The immunoreactive protein was detected using a chemical kit (Pierce Chemical Company, Rockford, IL). The tubulin and GAPDH were used as controls. Autoradiography is performed on the Alpha Innotech optical recording system (Alpha Innotech, CA, USA).

4.7. Tissue Histology. The kidneys soaked in $10 \%$ formalin were sectioned and then stained by hematoxylin. Images of the stained samples were taken using a microscope (Vectra 3, PerkinElmer).

4.8. Characterization. The products' shape investigation was performed on a scanning electron microscope (S4800, Hitachi, Tokyo, Japan). XRD (Shimadzu, Kyoto, Japan) was used to research the products' structure. The compositions of the obtained products were studied by FTIR (Shimadzu, Kyoto, Japan). Cell information was obtained by using CLSM (Leica TSC SP5 confocal unit, Buffalo Grove, IL, USA).

\section{ASSOCIATED CONTENT}

\section{SI Supporting Information}

The Supporting Information is available free of charge at https://pubs.acs.org/doi/10.1021/acsomega.0c05903.

Chemical formulae of main components of green TPs (PDF)

\section{AUTHOR INFORMATION}

\section{Corresponding Authors}

Zizhi Li - First Affiliated Hospital, Jinzhou Medical University, Jinzhou 121001, China; Email: lizizhi2010@ 126.com

Xiuli Ren - Jinzhou Medical University, Jinzhou 121001, China; Email: rxlrenxiuli@163.com

Zhenhua Chen - Jinzhou Medical University, Jinzhou 121001, China; orcid.org/0000-0002-0161-0236; Email: zhchen561@yahoo.com

\section{Authors \\ Linna Chang - Jinzhou Medical University, Jinzhou 121001, China \\ Yanan Hu - Jinzhou Medical University, Jinzhou 121001, China}

Complete contact information is available at:

https://pubs.acs.org/10.1021/acsomega.0c05903

\section{Notes}

The authors declare no competing financial interest.

\section{ACKNOWLEDGMENTS}

This work was supported by the National Natural Science Foundation of China (nos. 82072076 and 81771987), the Natural Science Foundation of Liaoning Province (no. 2019ZD-0812), the Innovative Talents Program of Liaoning Provincial Universities (no. LR2017075), and the Young Top Talents in Xing Liao Talents Program of Liaoning Province (XLYC1807183).

\section{REFERENCES}

(1) Sakhaee, K. Recent advances in the pathophysiology of nephrolithiasis. Kidney Int. 2009, 75, 585-595.

(2) Jeong, B. C.; Kim, B. S.; Kim, J. I.; Kim, H. H. Effects of Green Tea on Urinary Stone Formation: An in Vivo and in Vitro Study. J. Endourol. 2006, 20, 356-361.

(3) Wesson, J. A.; Worcester, E. M.; Kleinman, J. G. Role of anionic proteins in kidney stone formation: interaction between model anionic polypeptides and calcium oxalate crystals. J. Urol. 2000, 163, 1343-1348.

(4) Chung, J.; Granja, I.; Taylor, M. G.; Mpourmpakis, G.; Asplin, J. R.; Rimer, J. D. Molecular modifiers reveal a mechanism of pathological crystal growth inhibition. Nature 2016, 536, 446-450.

(5) Chen, Z.; Wang, C.; Zhou, H.; Sang, L.; Li, X. Modulation of calcium oxalate crystallization by commonly consumed green tea. CrystEngComm 2010, 12, 845-852.

(6) Backov, R.; Lee, C. M.; Khan, S. R.; Mingotaud, C.; Fanucci, G. E.; Talham, D. R. Calcium oxalate monohydrate precipitation at phosphatidylglycerol Langmuir monolayers. Langmuir 2000, 16, 6013-6019.

(7) Weaver, M. L.; Qiu, S. R.; Hoyer, J. R.; Casey, W. H.; Nancollas, G. H.; De Yoreo, J. J. Inhibition of calcium oxalate monohydrate growth by citrate and the effect of the background electrolyte. J. Cryst. Growth 2007, 306, 135-145.

(8) Jung, T.; Sheng, X.; Choi, C. K.; Kim, W.-S.; Wesson, J. A.; Ward, M. D. Probing crystallization of calcium oxalate monohydrate and the role of macromolecule additives with in situ atomic force microscopy. Langmuir 2004, 20, 8587-8596.

(9) Akyol, E.; Öner, M. Inhibition of calcium oxalate monohydrate crystal growth using polyelectrolytes. J. Cryst. Growth 2007, 307, 137-144.

(10) Wang, L.; Qiu, S. R.; Zachowicz, W.; Guan, X.; DeYoreo, J. J.; Nancollas, G. H.; Hoyer, J. R. Modulation of Calcium Oxalate 
Crystallization by Linear Aspartic Acid-Rich Peptides. Langmuir 2006, 22, 7279-7285.

(11) Sehgal, A.; Nasrul, S. I. Antilithogenic potential of green tea, oolong tea, and black tea. Pharmacogn. Res. 2020, 12, 92-94.

(12) Sheng, X.; Ward, M. D.; Wesson, J. A. Adhesion between Molecules and Calcium Oxalate Crystals: Critical Interactions in Kidney Stone Formation. J. Am. Chem. Soc. 2003, 125, 2854-2855.

(13) Chen, Z.; Wang, C.; Chen, J.; Li, X. Biocompatible, Functional Spheres Based on Oxidative Coupling Assembly of Green Tea Polyphenols. J. Am. Chem. Soc. 2013, 135, 4179-4182.

(14) Chen, Z.; Lv, X.; Zhao, M.; Zhang, P.; Ren, X.; Mei, X. Encapsulation of green tea polyphenol by $\mathrm{pH}$ responsive, antibacterial, alginate microgels used for minimally invasive treatment of bone infection. Colloids Surf., B 2018, 170, 648-655.

(15) Chen, G.; He, L.; Zhang, P.; Zhang, J.; Mei, X.; Wang, D.; Zhang, Y.; Ren, X.; Chen, Z. Encapsulation of green tea polyphenol nanospheres in PVA/alginate hydrogel for promoting wound healing of diabetic rats by regulating PI3K/AKT pathway. Mater. Sci. Eng., C 2020, 110, 110686.

(16) Zhang, P.; Li, D.; Chen, G.; Mei, X.; Zhang, J.; Chen, Z. Preparation of tea polyphenol- modified copper nanoclusters to promote the proliferation of MC3T3-E1 in high glucose microenvironment New. J. Chem. 2019, 43, 4082-4091.

(17) Zhou, K.; Ren, X.; Zhao, M.; Mei, X.; Zhang, P.; Chen, Z.; Zhu, $\mathrm{X}$. Promoting proliferation and differentiation of BMSCs by green tea polyphenols functionalized porous calcium phosphate. Regener. Biomater. 2018, 5, 35-41.

(18) Yi, Z.; Chen, G.; Chen, X.; Ma, X.; Cui, X.; Sun, Z.; Su, W.; Li, $X$. Preparation of Strong Antioxidative, Therapeutic Nanoparticles Based on Amino Acid-Induced Ultrafast Assembly of Tea Polyphenols. ACS Appl. Mater. Interfaces 2020, 12, 33550-33563.

(19) Yi, Z.; Chen, X.; Chen, G.; Deng, Z.; Tong, Q.; Sun, Z.; Ma, X.; Su, W.; Ma, L.; Ran, Y.; Li, X. General Nanomedicine Platform by Solvent-Mediated Disassembly/Reassembly of Scalable Natural Polyphenol Colloidal Spheres. ACS Appl. Mater. Interfaces 2020, 12, 37914-37928.

(20) Yi, Z.; Chen, G.; Chen, X.; Sun, Z.; Ma, X.; Su, W.; Deng, Z.; Ma, L.; Ran, Y.; Tong, Q.; Li, X. Modular Assembly of Versatile Nanoparticles with Epigallocatechin Gallate. ACS Sustainable Chem. Eng. 2020, 8, 9833-9845.

(21) Chen, X.; Yi, Z.; Chen, G.; Ma, X.; Su, W.; Cui, X.; Li, X. DOXassisted functionalization of green tea polyphenol nanoparticles for effective chemo-photothermal cancer therapy. J. Mater. Chem. B 2019, 7, 4066-4078.

(22) Zhang, H.; Yi, Z.; Sun, Z.; Ma, X.; Li, X. Functional nanoparticles of tea polyphenols for doxorubicin delivery in cancer treatment. J. Mater. Chem. B 2017, 5, 7622-7631.

(23) Rode, J.; Bazin, D.; Dessombz, A.; Benzerara, Y.; Letavernier, E.; Tabibzadeh, N.; Hoznek, A.; Tligui, M.; Traxer, O.; Daudon, M.; Haymann, J.-P. Daily Green Tea Infusions in Hypercalciuric Renal Stone Patients: No Evidence for Increased Stone Risk Factors or Oxalate-Dependent Stones. Nutrients 2019, 11, 256.

(24) Zhao, X.; Song, J.-L.; Yi, R.; Li, G.; Sun, P.; Park, K.-Y.; Suo, H. Comparison of Antioxidative Effects of Insect Tea and Its Raw Tea (Kuding Tea) Polyphenols in Kunming Mice. Molecules 2018, 23, 204.

(25) Sheng, X.; Ward, M. D.; Wesson, J. A. Crystal Surface Adhesion Explains the Pathological Activity of Calcium Oxalate Hydrates in Kidney Stone Formation. J. Am. Soc. Nephrol. 2005, 16, 1904-1908. (26) Tang, S.-L.; Zhao, Z.-W.; Liu, S.-M.; Wang, G.; Yu, X.-H.; Zou, J.; Wang, S.-Q.; Dai, X.-Y.; Fu, M.-G.; Zheng, X.-L.; Zhang, D.-W.; Fu, H.; Tang, C.-K. Pregnancy-Associated Plasma Protein-A Accelerates Atherosclerosis by Regulating Reverse Cholesterol Transport and Inflammation. Circ. J. 2019, 83, 515-523. 